\title{
Medición de desplazamientos nanométricos en polímeros piezoeléctricos usando método de descomposición en modos empíricos bivariados en patrones de speckle
}

\author{
Nanometric displacements measurement in piezoelectric polymers using bivariate empirical mode \\ decomposition method in speckle patterns \\ P. Etchepareborda*†1, F. Veiras ${ }^{\dagger \ddagger}$, A. Bianchetti* ${ }^{*}$ A. Federico ${ }^{\dagger}$, M. G. González $z^{\dagger \ddagger}$ \\ * INTI-Electrónica e Informática, Laboratorio de Técnicas Ópticas y Fotónicas (Latof) \\ Av. General Paz 5445, B1650WAB San Martín, Buenos Aires, Argentina \\ ${ }^{\dagger}$ Consejo Nacional de Investigaciones Científicas y Técnicas, (CONICET) \\ Godoy Cruz 2290, C1425FQB, Buenos Aires, Argentina \\ ${ }^{\ddagger}$ Universidad de Buenos Aires, Facultad de Ingeniería, \\ Grupo de Láser, Óptica de Materiales y Aplicaciones Electromagnéticas (GLOMAE) \\ Paseo Colón 850, C1063ACV, Buenos Aires, Argentina \\ ${ }^{1}$ pabloedinti.gob.ar \\ Recibido: 03/01/19; Aceptado: 14/02/19
}

\begin{abstract}
In this work we present an optical method for the direct determination of the piezoelectric coefficient of polymeric thin films. This is achieved through the measurement of nanometric mechanical displacements generated in the film when it is excited by low frequency harmonic electrical signals $(0.5 \mathrm{~Hz})$. The system is based on the temporal speckle pattern interferometry technique and the recovery of phase by using a bivariate empirical mode decomposition framework. The experimental scheme was used on a sample of vinylidene polyfluoride deposited on a glass substrate. The sample presents similar conditions to those found in the characterization of complex fluids by photoacoustic techniques. The measured value agrees with those obtained by other methods and with the value reported by the manufacturer.
\end{abstract}

Keywords: speckle; bivariate empirical mode descomposition; piezoelectric polymer.

Resumen- En este trabajo se presenta un método óptico para la determinación directa del coeficiente piezoeléctrico de películas delgadas de material polimérico. Esto se logra a través de la medición de desplazamientos mecánicos nanométricos generados en el film cuando es excitado con señales eléctricas armónicas de baja frecuencia $(0.5 \mathrm{~Hz})$. E sistema está basado en la inteferometría temporal de patrones de speckle y en la recuperación de fase por descomposición en modos empíricos bivariada. El esquema experimental fue usado sobre una muestra de polifluoruro de vinilideno depositada sobre un substrato de vidrio que presenta condiciones de contorno similares a las que se encuentran en experiencias de caracterización de fluídos complejos por técnicas fotoacústicas. El valor medido concuerda con aquellos obtenidos por otros métodos y con el reportado por el fabricante.

Palabras clave: speckle; descomposición de modos empíricos bivariados; polímero piezoeléctrico.

\section{INTRODUCCIÓN}

En el campo del diseño y fabricación de MEMS y MOEMS existe un gran interés en la caracterización de materiales que sirvan para mejorar los procesos de ensamblado de microcomponentes y aumentar las funcionalidades de los dispositivos, especialmente en cuanto a la capacidad de integración, de sensado, actuación y adaptabilidad al medio [1]. Los polímeros piezoeléctricos, como el polifluoruro de vinilideno (PVDF) y sus copolímeros de estructura más cristalina sintetizados mediante el agregado de monómeros, ofrecen una significativa versatilidad para su utilización en diversas aplicaciones debido a sus propiedades físicas. Estos materiales son flexibles, están disponibles como películas delgadas, tienen un gran ancho de banda acústica, y sus valores de impedancia acústica están próximos al del agua y los tejidos biológicos (a las frecuencias de interés) [2]. Estas propiedades los hacen muy útiles para la manipulación controlada de muestras biológicas y otras aplicaciones biomédicas [3]. Otros campos de aplicación de los polímeros piezoeléctricos son la ecografía, tomografía fotoacústica, hidrófonos, análisis de vibraciones, ensayos no destructivos en materiales, sensores de presión y micrófonos [4].

En la actualidad se investiga la manera de mejorar las propiedades piezoeléctricas de los polímeros a través del dopado con nanocompuestos [5], [6]. Por tal motivo, es de particular interés contar con un sistema confiable para medir las propiedades electromecánicas de estos nuevos polímeros, especialmente el coeficiente piezoeléctrico que relaciona la deformación del material con la diferencia de potencial aplicada. La magnitud típica de piezoelectricidad en estos materiales es muy baja en comparación con otros componentes piezoeléctricos (entre 10 y 30 veces menor que la correspondiente a piezoeléctricos cerámicos), ha- 
ciendo que la tarea de caracterización requiera métodos de alta sensibilidad. La determinación de este parámetro suele hacerse en forma indirecta mediante la medición de la impedancia eléctrica a frecuencias cercanas a la primera resonancia mecánica. La resonancia poco acentuada de los polímeros hace que la determinación de los parámetros a partir de una técnica de ajuste de los datos al modelo físico posea una incerteza considerablemente alta. Esto hace que continuamente se propongan nuevos métodos de medición más precisos [7]-[9].

En este trabajo se presenta un método óptico que permite la determinación directa del coeficiente piezoeléctrico midiendo los desplazamientos mecánicos generados por la aplicación de excitaciones eléctricas armónicas de baja frecuencia $(<1 \mathrm{~Hz})$. El sistema está basado en la inteferometría temporal de patrones de speckle y en la recuperación de fase por descomposición en modos empíricos bivariada. Para verificar su funcionamiento se usó una muestra de PVDF depositada sobre un substrato de vidrio que presenta condiciones de contorno similares a las que se encuentran en experiencias de caracterización de fluídos complejos por técnicas fotoacústicas [10]-[13].

El trabajo está organizado de la siguiente manera. En la sección II se describe el método óptico utilizado para medir los desplazamientos de la película delgada de PVDF. En las secciones III y IV se detalla el esquema experimental implementado y los resultados obtenidos, respectivamente. Finalmente, en la sección $\mathrm{V}$, se presentan las conclusiones de este trabajo.

\section{RECUPERACIÓN DE FASE MEDIANTE BIVEMD}

La distribución de intensidad en una secuencia de imágenes de interferometría temporal de patrones de speckle (TSPI), donde la adquisición de imágenes se efectúa en forma periódica durante el proceso de variaciones en la muestra, se describe a través de la siguiente ecuación:

$$
I_{i}(x, y, t)=I_{F}(x, y, t)+I_{M}(x, y, t) \cos \left(\phi_{d}(x, y, t)\right)
$$

La intensidad resultante es la superposición de una intensidad de fondo $I_{F}$ y el coseno de la diferencia de fase $\phi_{d}=\phi_{o}-\phi_{r}$ con una amplitud modulada por las variaciones lentas y aleatorias de la intensidad de modulación $I_{M}$. Las variaciones temporales de $I_{F}$ y $I_{M}$ se suponen siempre en soportes de menor frecuencia que las propias del coseno de $\phi_{d}$. La fase de referencia $\phi_{r}$ suele corresponder a la portadora temporal y admite variaciones espaciales. La fase de objeto $\phi_{o}$ resulta de la fase impuesta por la superficie rugosa del objeto y por su estructura, siendo que esta última posee las variaciones micro y nanométricas a medir. El objetivo de los métodos de procesamiento digital para TSPI es explotar distintas propiedades de $I_{i}(t)$ para aislar el término de intensidad con modulación removiendo la presencia de la intensidad de fondo. Luego, en el que caso de utilizar portadora temporal el procedimiento debe caracterizar las variaciones de fase que se apartan de la portadora. El método más usual de recuperación de fase en TSPI se basa en el uso de la FFT (Fast Fourier Transform) para realizar un procesamiento temporal [14].
La frecuencia central y el ancho de banda del filtro que se utiliza en el método de FFT deben ser apropiadamente ajustados para cada secuencia de imágenes considerada, o incluso localmente para cada zona de píxeles, de acuerdo a la magnitud máxima de variaciones de fase y la relación señal a ruido particulares. Esta tarea no es trivial y suele aminorarse en complejidad con la implementación de la transformada ventaneada de Fourier (WFT por sus siglas en inglés) o la transformada wavelet contínua (CWT) [15]. La robustez frente a ruido de estos métodos resulta superior al de FFT, en particular si el espectro de ruido se superpone a la banda de frecuencias de la señal modulada. Además, estos enfoques han sido generalizados para el análisis de las secuencias de imágenes con el objetivo de utilizar información de píxeles vecinos en la demodulación temporal de fase, particularmente en píxeles con baja intensidad de modulación propensos a desenganches de fase [16]. Sin embargo, estos análisis acumulan errores de recuperación de fase obteniendo grandes desviaciones en el resultado final, especialmente cuando se reduce la cantidad de bandas de frecuencia o escalas de descomposición con el objetivo de reducir la complejidad de cálculo.

La modulación de una portadora compleja por la señal temporal de intensidad en la ecuación de interferometría con dos haces representada en la ec. 1, obtiene una señal compleja que puede ser filtrada para recuperar la información de fase. La extensión bivariada del algoritmo basado en la descomposición en modos empíricos, conocida por las siglas EMD (en inglés, Empirical Mode Decomposition) ofrece una alternativa adaptiva para implementar dicho filtrado [17]. Este procedimiento permite obtener un método simplificado de recuperación de fase. A continuación se describen los detalles de este método para ser usado en TSPI.

La modificación inicial que se propone para la ecuación interferométrica consiste en restar la intensidad de fondo $I_{F}$ a la intensidad observada $I_{i}$ y la multiplicación por el factor de fase de la portadora compleja $\exp \left(j \phi_{r}(t)\right)$. En resumen,

$\left(I_{i}-I_{F}\right) \exp \left(j \phi_{r}\right)=\frac{I_{M}}{2}\left\{\exp \left(j \phi_{o}\right)+\exp \left[j\left(2 \phi_{r}-\phi_{o}\right)\right]\right\}$

donde las dependencias espacio temporales de las variables se omitieron para mayor claridad. En el lado derecho de esta ecuación, se destaca una señal analítica de tipo AMFM con dos términos distintivos. El primer término contiene únicamente a la fase objeto $\phi_{o}$ que es la que se quiere determinar. El segundo término complejo tiene como fase a $\psi:=2 \phi_{r}-\phi_{o}$, de más rápidas variaciones. Por lo tanto, es fácil distinguir dos escalas de tiempo características distintas entre los términos, dado que las variaciones de la fase objeto son notablemente más lentas que las de $\psi$. Es importante notar que la evolución temporal de $\phi_{o}$ es altamente determinante del rendimiento del método de recuperación de fase ya que si la fase objeto posee variaciones rápidas se puede producir un solapamiento entre las escalas de tiempofrecuencia características.

En el plano complejo, la evolución temporal de las partes real e imaginarias de la serie temporal bivariada caracterizada por la ec. $2, z(t):=\left[I_{i}(t)-I_{F}(t)\right] \exp \left[j \phi_{r}(t)\right]$, se asimilan a las coordenadas cartesianas de un punto que se 

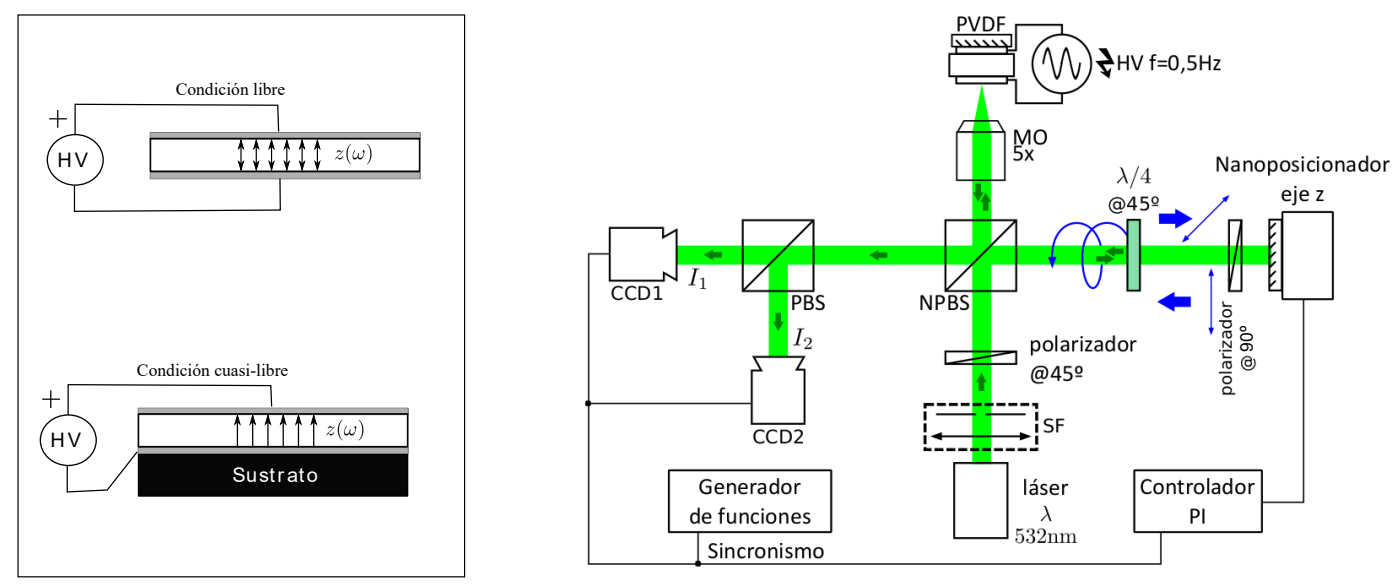

Fig. 1. Izquierda: esquemas de la construcción de transductor de PVDF en las condiciones libre (arriba) y con sustrato o cuasi-libre (abajo). Derecha: esquema óptico basado en SQHI para la medición de desplazamientos en una lámina de PVDF.

mueve en un plano. De la misma manera, los dos términos mencionados en la ec. 2 se representan en este espacio bidimensional como la superposición de una rotación rápida dada por $\psi(t)$ y otra más lenta correspondiente a $\phi_{o}(t)$. Se propone, por lo tanto, un análisis bivariado que descompone a $z(t)$ en términos rotacionales. El algoritmo EMD bivariado (BivEMD) propuesto en [17] adapta el concepto de oscilaciones utilizado en EMD al caso bivariado de las rotaciones. De esta manera, los dos términos rotacionales superpuestos pueden ser efectivamente identificados y separados mediante la aplicación de BivEMD a la señal $z(t)$ si previamente se consigue una estimación de $I_{F}(t)$. Es importante notar que esta técnica bivariada no debe ser confundida con EMD bidimensional, la cual realiza una búsqueda de modos empíricos en imágenes.

Por construcción, el algoritmo BivEMD descompone adaptivamente a $z(t)$ en un conjunto de modos de oscilación o funciones de modo intrínsecas bivariadas (BIMF) con rotaciones de diferentes escalas de tiempo y un residuo de tendencia que es no rotacional [18]-[20]. El esquema iterativo de BivEMD obtiene primero la BIMF de rotaciones más rápidas y luego extrae progresivamente las BIMF de rotación más pausada. Este mecanismo de separación empírico permite seleccionar y conservar la suma de BIMFs correspondientes al término rotacional dado por $\phi_{o}(t)$ en la ec. 2. Note que la selección de BIMF a considerar es más simple que la elección del ancho de banda y la frecuencia central del filtro en el método por FFT. En este trabajo, siempre se descartó la primera BIMF y se extrajeron para el análisis la segunda, o incluso, la tercera BIMF. Consecuentemente, la fase objeto se recupera por medio de la función arcotangente de dos argumentos y la evolución temporal de fase de cada píxel del sensor CCD se obtiene a través de un procedimiento unidimensional de desenvolvimiento de fase. Más detalles sobre este método y su algoritmo se encuentran en [18]-[20].

\section{MATERiAles Y MÉTOdos}

Una configuración muy utilizada para realizar sensores de banda ancha consiste en metalizar electrodos (usualmente aluminio) sobre una película de material piezoeléctrico [21]. En el caso del PVDF, durante su fabricación, la película es estirada y luego polarizada mediante la aplicación de un campo eléctrico en la dirección perpendicular de la misma. Usualmente se adopta una terna ortogonal de referencia, donde la dirección de estiramiento en el plano del film se toma como eje de referencia 1 , y la dirección de polarización como eje de referencia 3 (normal a la película). Si se aplica una diferencia de potencial entre los electrodos con las condiciones de contorno mostradas en los gráficos de la Fig. 1 (simetría en el plano del film), la película delgada de PVDF puede ser modelada como un transductor 1-D. En condición isotérmica y excitación armónica de frecuencia $\omega$, el sistema de ecuaciones constitutivas para un film de polímero piezoeléctrico de área $A$ y espesor $\delta$ es [8]:

$$
\begin{aligned}
I(\omega) & =\frac{j \omega A \varepsilon_{F}(\omega)}{\delta} V(\omega)+j \omega d_{33}(\omega) F(\omega) \\
U(\omega) & =j \omega d_{33}(\omega) V(\omega)+\frac{j \omega \delta s_{33}(\omega)}{A} F(\omega)
\end{aligned}
$$

donde $I, V, F$ y $U$ son las variables medibles de corriente, tensión eléctrica, fuerza y velocidad integradas sobre la superficie de la muestra, respectivamente. Conjuntamente, $\varepsilon_{F}, d_{33}$ y $s_{33}$ son los parámetros intensivos característicos de la muestra: la permitividad eléctrica en condición de esfuerzo mecánico nulo, el coeficiente piezoeléctrico en la dirección del campo de polarización, y la compliancia elástica a campo eléctrico constante, respectivamente. En la condición de esfuerzo mecánico cuasi nulo o condición casi libre es posible relacionar de forma sencilla al máximo desplazamiento $\hat{z}(\omega)$ de la superficie ante una diferencia de tensión aplicada entre los electrodos según [4]:

$$
\hat{z}(\omega)=d_{33}(\omega) V(\omega)
$$

En la Fig. 1 (derecha) se muestra el esquema óptico propuesto, utilizado para medir muy bajos desplazamientos fuera del plano en una lámina de PVDF del fabricante Piezotech S.A.S. de $25 \mu \mathrm{m}$ de espesor montada sobre un sustrato rígido (vidrio BK7 de $1 \mathrm{~mm}$ de espesor). Como se muestra en [22], para remover la influencia del término de modulación de intensidad de los interferogramas y obtener una mayor precisión, los interferómetros heterodinos de 
fase rotada en $\pi / 2$ en simultáneo (SQHI de sus siglas en inglés) resultan muy convenientes. Asimismo, los resultados obtenidos en [19] muestran que es posible reducir aún más los niveles de ruido mediante la combinación de las técnicas SQHI y BivEMD.

Se implementó un esquema SQHI con una magnificación modificada por el agregado de un objetivo de microscopio de $5 x$. Este esquema consiste en un interferómetro de TwymanGreen con la adquisición en simultáneo de las imágenes con fase rotada en los sensores CCD1 y CCD2. Esto se obtiene mediante la utilización de un interferómetro con luz polarizada (Fig. 1). El haz principal se polariza mediante un polarizador a 45 grados y se reparte en partes aproximadamente iguales a través del prisma no polarizado (NPBS) hacia la muestra de PVDF y hacia el espejo montado sobre el nano-posicionador. La rama que se desvía hacia el espejo atraviesa en una primera pasada una lámina retardadora a 45 grados que no altera el estado de polarización del haz ya que el eje de la lámina coincide con la polarización lineal del haz. Este pasa por un polarizador horizontal respecto del plano de la mesa y se refleja en el espejo montado sobre el nano-posicionador, retornando hacia la lámina retardadora polarizado horizontalmente. Como la lámina se encuentra a 45 grados de la dirección horizontal, el haz emerge de la lámina de cuarto de onda, en esta segunda pasada, con polarización circular. Luego el NPBS recombina los haces provenientes de la muestra de PVDF y del espejo. El primero con polarización lineal a 45 grados se descompone (mediante el PBS) en 2 campos que oscilan en fase, uno polarizado verticalmente y otro polarizado horizontalmente. En cambio, el haz de referencia, con polarización circular, se descompone en dos campos que oscilan con una diferencia de fase de $\pi / 2$, uno polarizado verticalmente y otro polarizado horizontalmente.

En esta descomposición se tienen dos haces linealmente polarizados cuya intensidad tiene la información correspondiente interferogramas heterodinos desfasados en $\pi / 2$ que son proyectados sobre los sensores CCD1 y CCD2.

Para esta experiencia se utilizó una fuente láser B\&W Tek, Inc. de $532 \mathrm{~nm}$ y la modulación temporal se realizó mediante el movimiento de un espejo con un nanoposicionador en eje $\mathrm{z}$ modelo PI P-611.Z y su controlador PI E-625, obteniendo resolución en el movimiento de $0.2 \mathrm{~nm}$ y un error de linealidad de $0.1 \%$. Se aplicaron señales de tensión sinusoidales de frecuencia $0.5 \mathrm{~Hz}$ con diversas amplitudes mediante la programación de la fuente de tensión controlada del electrómetro KEITHLEY 6517A. Las señales de sincronismo de las cámaras y el controlador del nanoposicionador fueron establecidas por un generador de funciones arbitrarias modelo Tabor Electronics WS8102.

Un beneficio de usar este arreglo es que si ambos interferogramas se superponen adecuadamente y se restan entre sí, se obtiene una imagen cuyos valores corresponden solamente a la modulación de intensidad dada por la interferencia, eliminando los efectos indeseados de las intensidades de fondo de cada uno de los haces involucrados.

\section{RESUltAdos EXPERIMENTALES}

En la Fig. 2 se muestran algunas de las evoluciones de desplazamiento obtenidas. La Fig. 2 (a) corresponde a la

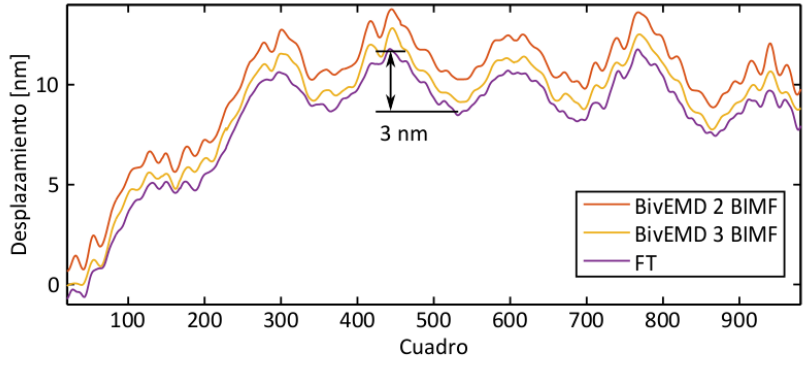

(a)

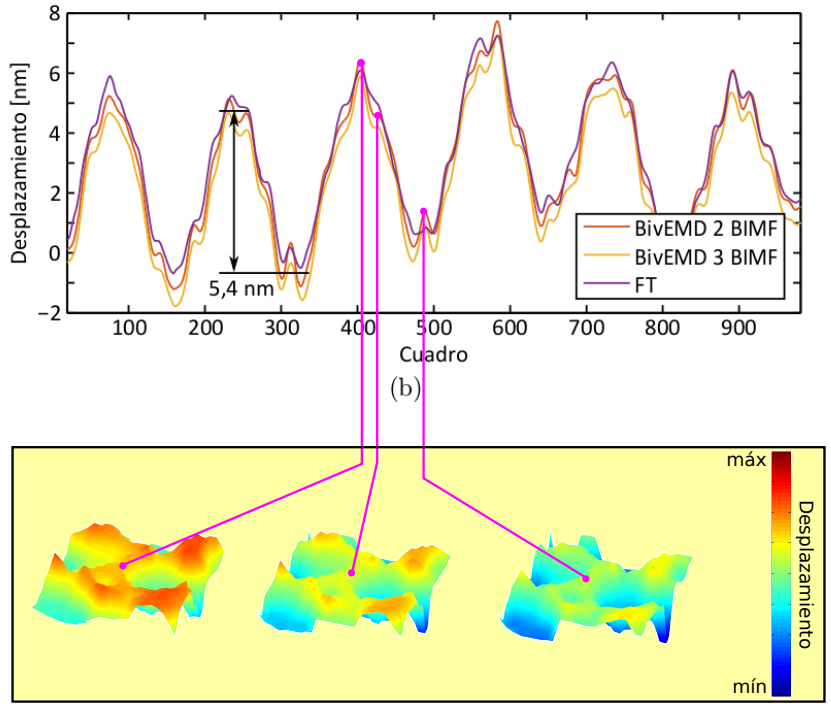

(c)

Fig. 2. Evolución media de fase medida en una zona de la lámina de PVDF cuando se aplica una excitación de tensión con forma sinusoidal de amplitud (a) $140 \mathrm{~V}$, y (b) $300 \mathrm{~V}$. Se muestran los resultados obtenidos mediante la reconstrucción de fase mediante los métodos de FT y BivEMD suprimiendo 2 o 3 BIMFs. Se muestran valores pico a pico de los desplazamientos. (c) Imágenes captadas y procesadas usando BivEMD para una tensión de 300 $\mathrm{V}$.

situación de aplicar $140 \mathrm{~V}$ como valor pico de tensión y la Fig. 2 (b) corresponde a 300 V. Se muestran cotas para facilitar la observación de los valores pico a pico de los desplazamientos. Las evoluciones mostradas corresponden a las técnicas de reconstrucción de fase en TSPI usando FFT y BivEMD con extracción de 2 y 3 BIMFs. Se observa una gran similaridad en los resultados de los tres métodos. Sin embargo, la aplicación del método BivEMD con la extracción de 2 BIMFs en los resultados de la excitación de $300 \mathrm{~V}$ muestra variaciones repetitivas sumadas a la señal fundamental que son del doble de amplitud que las observadas para las otras curvas. El desvío estándar de las señales luego de ser filtradas para atenuar las componentes de frecuencias cercanas a la señal de excitación de la muestra fueron $(0,49 ; 0,29 ; 0,22)$ radianes para BivEMD 2 BIMF, 3 BIMF y FT, respectivamente. Las observaciones realizadas durante la configuración del controlador que impone los desplazamientos en el espejo para generar la portadora temporal permiten asociar estas variaciones a los errores experimentales en la conformación de la portadora. Los métodos FT y BivEMD con extracción de 3 BIMFs realizan un filtrado de estas variaciones ocultando dichos detalles.

Debido al bajo nivel del coeficiente piezoeléctrico en la dirección del campo de polarización $\left(d_{33}\right)$ las distintas 


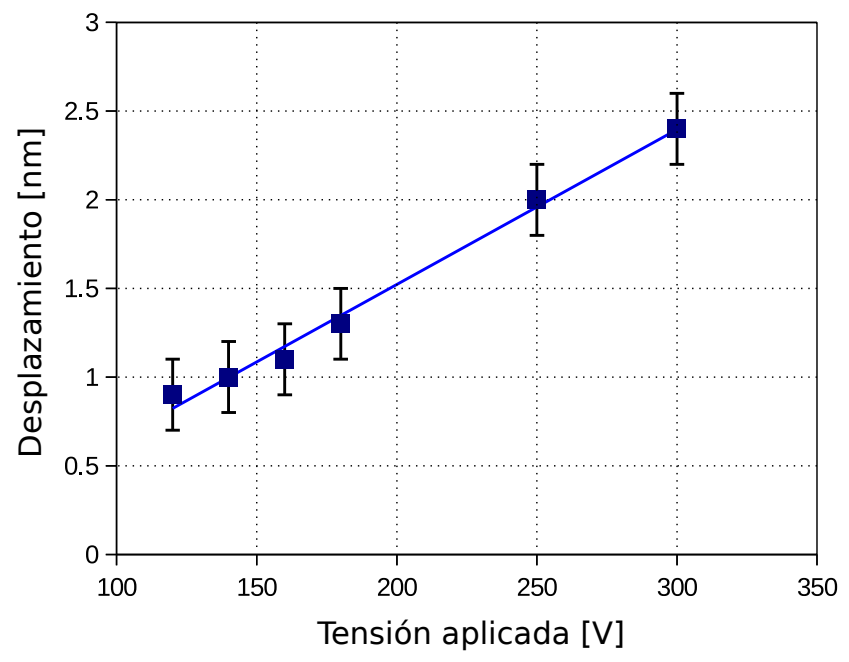

Fig. 3. Desplazamiento pico medio medido sobre la superficie del film de PVDF en función de la tensión aplicada con frecuencia de $0.5 \mathrm{~Hz}$.

tensiones aplicadas de $120 \mathrm{~V}$ a $300 \mathrm{~V}$ produjeron desplazamientos de amplitudes entre $0.9 \mathrm{~nm}$ y $2.4 \mathrm{~nm}$. La reducida magnitud de estos desplazamientos obliga el uso de técnicas de medición altamente sensibles. Como puede observarse en la Fig. 2 (b), para tensiones de $300 \mathrm{~V}$, es posible estimar la amplitud de estos desplazamientos sin mayores dificultades. Esta situación empeora para tensiones inferiores, resultando más difícil distinguir la amplitud de la señal asociada a los desplazamientos del PVDF. De hecho, en la Fig. 2 (a) se observan variaciones nanométricas muy lentas superpuestas con la señal sinusoidal de la excitación. Además, la Fig. 2 (c) muestra que las variaciones espaciales de altura medidas tienen una textura cuya magnitud es similar o superior a las variaciones debidas a la deformación media analizada. A partir de las mediciones para distintas tensiones (ver Fig. 3) se pudo estimar el coeficiente piezoeléctrico mediante el uso de la ec. 5 , resultando $d_{33}=9 \mathrm{pm} / \mathrm{V}$.

Es importante notar que el resultado obtenido es del mismo orden de magnitud que el reportado por el fabricante $(15 \mathrm{pm} / \mathrm{V} \pm 20 \%)$. Asimismo, este valor de baja frecuencia $(0.5 \mathrm{~Hz})$ también es consistente con la mediciones realizadas sobre el mismo lote de PVDF con dos métodos distintos (método indirecto por espectro dieléctrico y método directo óptico por interferometría heterodina) reportadas en [7].

\section{CONCLUSiOnes}

En este trabajo se presentó un método óptico que permite medir desplazamientos armónicos de baja frecuencia en películas delgadas de polímeros piezoeléctricos. Se midió una muestra de PVDF despositada sobre un substrato de vidrio y se implementó un sistema de detección basado en la interferometría temporal de patrones de speckle y recuperación de fase mediante el método BivEMD.

Se logró medir el coeficiente piezoeléctrico de baja frecuencia. Este dato resulta de gran interés debido a que es el valor representativo en el rango audible de frecuencias $(<$ $10 \mathrm{kHz}$ ); lejos de la primera relajación dieléctrica [8]. El valor obtenido concuerda con los valores reportados por el fabricante y con otros métodos de medición [7] aplicados sobre muestras provenientes del mismo lote.
La técnica empleada permite detectar las deformaciones localizadas sobre la superficie en el caso de adquirir imágenes que cubran a la totalidad de la muestra. De esta manera, se posibilita una evaluación tridimensional del transductor de PVDF, mientras que los métodos directos e indirectos usuales [7], [8] de caracterización de materiales piezoeléctricos utilizan modelos unidimensionales.

\section{AGRADECIMIENTOS}

Este trabajo fue apoyado por los subsidios de la Universidad de Buenos Aires (UBACyT 20020170200232BA) y de la ANPCyT (PICT 2016-2204).

\section{REFERENCIAS}

[1] K. S. Ramadan, D. Sameoto, and S. Evoy, "A review of piezoelectric polymers as functional materials for electromechanical transducers," Smart Materials and Structures, vol. 23, p. 033001, 2014.

[2] L. C. Brazzano, P. Sorichetti, G. Santiago, and M. González, "Broadband dielectric characterization of piezoelectric poly (vinylidene fluoride) thin films between $278 \mathrm{k}$ and $308 \mathrm{k}$," Polymer Testing, vol. 32, pp. 1186-1191, 2013.

[3] Y. Shen, N. Xi, K. W. Lai, and W. J. Li, "A novel pvdf microforce/force rate sensor for practical applications in micromanipulation," Sensor Rev., vol. 24, pp. 274-283, 2004.

[4] A. F. Vidal, L. C. Brazzano, C. Matteo, P. Sorichetti, and M. G. González, "Parametric modeling of wideband piezoelectric polymer sensors: design for optoacoustic applications," Rev. Sci. Instrum. vol. 88, p. 095004, 2017.

[5] X. Cao, J. Ma, X. Shi, and Z. Ren, "Effect of tio 2 nanoparticle size on the performance of pvdf membrane," Appl. Surf. Sci., vol. 253, pp. 2003-2010, 2006.

[6] J. S. Dodds, F. N. Meyers, and K. J. Loh, "Piezoelectric characterization of pvdf-trfe thin films enhanced with zno nanoparticles," IEEE Sens. J., vol. 12, pp. 1889-1890, 2012.

[7] L. M. Riobo, F. E. Veiras, M. G. Gonzalez, M. T. Garea, and P. A. Sorichetti, "High-speed real-time heterodyne interferometry using software-defined radio," Appl. Opt., vol. 57, pp. 217-224, 2018.

[8] M. Gonzalez, P. Sorichetti, L. C. Brazzano, and G. Santiago, "Electromechanical characterization of piezoelectric polymer thin films in a broad frequency range," Polymer Testing, vol. 37, pp. 163-169, 2014.

[9] V. Rathod, D. R. Mahapatra, A. Jain, and A. Gayathri, "Characterization of a large-area pvdf thin film for electro-mechanical and ultrasonic sensing applications," Sensor. Actuat. A-Phys., vol. 163, pp. 164-171, 2010

[10] M. G. Gonzalez, E. Acosta, and G. D. Santiago, "Determination of the thermal boundary conductance of gold nanoparticles in aqueous solution using a method based on nanobubble generation," Appl. Opt., vol. 57, pp. 6229-6232, 2018.

[11] L. Cusato, M. Estevez, M. G. Gonzalez, and G. D. Santiago, "Caracterización de líquidos por técnica fotoacústica: Estudio de sensores piezoeléctricos de banda ancha," Elektron, vol. 1, pp. 8-15, 2017.

[12] E. Acosta, M. G. Gonzalez, P. A. Sorichetti, and G. D. Santiago, "Laser-induced bubble generation on a gold nanoparticle: A nonsymmetrical description," Phys. Rev. E, vol. 92, p. 062301, 2015.

[13] M. G. Gonzalez, X. Liu, R. Niessner, and C. Haisch, "Lead ion detection in turbid media by pulsed photoacoustic spectrometry based on dissolution of gold nanoparticles," Sensors and Actuators B: Chemical, vol. 150, pp. 770-773, 2010.

[14] L. Seminara, M. Capurro, P. Cirillo, G. Cannata, and M. Valle, "Phase measurement in temporal speckle pattern interferometry using the fourier transform method with and without a temporal carrier," Opt. Comm., vol. 217, pp. 141-149, 2003.

[15] Y. Fu, C. J. Tay, C. Quan, and H. Miao, "Wavelet analysis of speckle patterns with a temporal carrier," Applied optics, vol. 44, no. 6, pp. 959-965, 2005.

[16] A. Federico and G. H. Kaufmann, "Robust phase recovery in temporal speckle pattern interferometry using a $3 \mathrm{~d}$ directional wavelet transform," Optics letters, vol. 34, no. 15, pp. 2336-2338, 2009.

[17] G. Rilling, P. Flandrin, P. Gonalves, and J. Lilly, "Bivariate empirical mode decomposition," IEEE Signal Process. Lett., vol. 14, pp. 936939, 2007.

[18] P. Etchepareborda, A. Bianchetti, A. L. Vadnjal, A. Federico, and G. H. Kaufmann, "Simplified phase-recovery method in temporal speckle pattern interferometry," Applied optics, vol. 53, no. 30, pp. 7120-7128, 2014. 
Revista elektron, Vol. 3, No. 1, pp. $52-57$ (2019)

[19] P. Etchepareborda, A. Bianchetti, F. E. Veiras, A. L. Vadnjal, A. Federico, and G. H. Kaufmann, "Comparison of real-time phasereconstruction methods in temporal speckle-pattern interferometry," Applied optics, vol. 54, no. 25, pp. 7663-7672, 2015.

[20] P. Etchepareborda, "Investigación e implementación de técnicas de procesamiento de señales e imágenes para la inspección óptica de microsistemas," Ph.D. dissertation, Univ. de Buenos Aires, Buenos Aires, Argentina, 2016.

[21] M. Gonzalez, P. Sorichetti, and G. Santiago, "Reducing the capacitance of piezoelectric film sensors," Rev. Sci. Instrum., vol. 87, p. 045003, 2016

[22] A. Bianchetti, F. E. Veiras, P. Etchepareborda, A. L. Vadnjal, A. Federico, and G. H. Kaufmann, "Amplitude and phase retrieval in simultaneous $\pi / 2$ phase-shifting heterodyne interferometry using the synchrosqueezing transform," Applied optics, vol. 54, no. 8, pp. 2132 2140, 2015. 